TABLE 2. Values of $k$ and corresponding values of $r$

\begin{tabular}{|c|c|c|c|c|c|c|c|}
\hline$k$ & $r$ & $k$ & $r$ & $k$ & $r$ & $k$ & $r$ \\
\hline 3 & 1 & 55 & 1 & 109 & 2 & 165 & 2 \\
\hline 4 & 1 & 56 & 1 & 111 & 1 & 167 & 1 \\
\hline $\begin{array}{l}5 \\
7\end{array}$ & $\begin{array}{l}2 \\
1\end{array}$ & $\begin{array}{l}56^{*} \\
57\end{array}$ & $\begin{array}{l}2 \\
2\end{array}$ & $\begin{array}{l}113 \\
115\end{array}$ & ${ }_{4}^{2}$ & $\begin{array}{l}168 \\
168^{*}\end{array}$ & 2 \\
\hline & $\begin{array}{l}1 \\
2\end{array}$ & & 1 & $\begin{array}{l}110 \\
116\end{array}$ & $\begin{array}{l}4 \\
1\end{array}$ & 172 & 2 \\
\hline $8^{*}$ & 1 & 60 & 2 & 119 & 1 & 177 & 2 \\
\hline 11 & 1 & 61 & 2 & 120 & 1 & 179 & 2 \\
\hline 12 & 2 & 65 & 2 & $120^{*}$ & 2 & 181 & 2 \\
\hline 13 & 2 & 68 & 1 & 124 & 2 & 183 & 1 \\
\hline 15 & & 69 & 2 & 127 & 2 & 184 & 2 \\
\hline 17 & 2 & 71 & 1 & 129 & 2 & $184^{*}$ & 2 \\
\hline $\begin{array}{l}19 \\
?\end{array}$ & 2 & $\begin{array}{l}73 \\
76\end{array}$ & 2 & 131 & 1 & $\begin{array}{l}185 \\
101\end{array}$ & \\
\hline & $\begin{array}{l}1 \\
2\end{array}$ & 76 & $\frac{2}{7}$ & 132 & 1 & 191 & $\frac{1}{2}$ \\
\hline $\begin{array}{l}21 \\
23\end{array}$ & 1 & 79 & 1 & 133 & 2 & 193 & 2 \\
\hline 23 & & 79 & & 136 & 2 & 195 & 2 \\
\hline 24 & 1 & 83 & 1 & $136^{*}$ & 1 & 199 & 1 \\
\hline $24^{*}$ & 2 & 84 & 1 & 137 & 2 & 201 & 2 \\
\hline 28 & 2 & $\begin{array}{l}85 \\
87\end{array}$ & 2 & 139 & 2 & 203 & 2 \\
\hline 29 & 2 & 87 & 1 & 140 & 2 & 204 & 2 \\
\hline 31 & 1. & $88^{*}$ & 2 & 141 & 2 & 205 & 2 \\
\hline 33 & 2 & 89 & 2 & 143 & 1 & 209 & 2 \\
\hline 35 & 1 & 91 & 3 & 145 & 2 & 211 & 3 \\
\hline 37 & 2 & 92 & ${ }_{2}^{2}$ & 149 & ${ }_{1}^{2}$ & $\begin{array}{l}212 \\
213\end{array}$ & 1 \\
\hline $\begin{array}{l}39 \\
40\end{array}$ & $\begin{array}{l}1 \\
2\end{array}$ & $\begin{array}{l}93 \\
95\end{array}$ & ${ }_{1}^{2}$ & $\begin{array}{l}151 \\
152\end{array}$ & 1 & $\begin{array}{l}213 \\
215\end{array}$ & 6 \\
\hline & & & & 152 & 1 & 215 & 1 \\
\hline $40^{*}$ & 1 & 97 & 2 & $152^{*}$ & 9 & 217 & 2 \\
\hline 41 & 2 & 101 & 2 & 155 & 2 & 219 & 2 \\
\hline 44 & 2 & 103 & 1 & 156 & 2 & 220 & 2 \\
\hline 47 & 1 & 104 & 2 & 157 & 2 & 221 & 2 \\
\hline 51 & 2 & $104^{*}$ & 1 & 159 & 1 & 223 & 2 \\
\hline 52 & 2 & 105 & 2 & 161 & 2 & 227 & 2 \\
\hline 53 & 3 & 107 & 2 & 164 & 1 & & \\
\hline
\end{tabular}

TABLE 3. Talues of $k$ and corresponding combinations and values of $r$

\begin{tabular}{|c|c|c|}
\hline$k$ & Combination & $r$ \\
\hline $\begin{array}{r}43 \\
67 \\
88 \\
123 \\
148 \\
173 \\
187 \\
188 \\
197\end{array}$ & $\begin{array}{l}\left(1+2^{-s}\right)\left(1+3^{-s}\right) L(s, \chi) \\
\left(1+2^{-s}\right)\left(1+3^{-s}\right)\left(1+5^{-s}\right) L(s, \chi) \\
\left(1+3^{-s}\right) L(s, \chi) \\
\left(1+2^{-s}\right)\left(1+5^{-s}\right) L(s, \chi) \\
\left(1+3^{-s}\right)\left(1+5^{-s}\right)\left(1+7^{-s}\right) L(s, \chi) \\
\left(1+2^{-s}\right)\left(1+3^{-s}\left(1+5^{-s}\right) L(s, \chi)\right. \\
\left(1+2^{-s}\right)\left(1+3^{-s}\right)\left(1+5^{-s}\right) L(s, \chi) \\
\left(1+3^{-s}\right) L(s, \chi) \\
\left(1+2^{-s}\right) L(s, \chi)\end{array}$ & $\begin{array}{l}2 \\
3 \\
4 \\
2 \\
3 \\
4 \\
3 \\
3 \\
2\end{array}$ \\
\hline
\end{tabular}

\section{References}

[1] J. Barkley Rosser, Real roots of Dirichlet L-series, Bul. Am. Math. Soc. 55, 906 to 913 (1949).

[2] E. Landau, Handbuch der Lehre von der Verteilung der Primzahlen, 1, Leipzig, Teubner, 1909.

[3] S. Chowla, Note on Dirichlet's L-functions, Acta Arithmetica, 1, 113 to 114 (1935).

[4] H. Heilbronn, On real characters, Acta Arithmetica, 2, 212 to 213 (1937).

[5] E. T. Whittaker and G. N. Watson, A course of modern analysis, American Edition (The Macmillan Co., New York, N. Y., 1947).

[6] E. Landau, Vorlesungen über zahlentheorie, 1, Leipzig, Hirzel, 1927.

[7] S. Chowla and A. Selberg, On Epstein's Zeta Function (I), Proc. Nat. Acad. of Sciences, 35, 371 to 374 (1949)

[8] H. Heilbronn, On Dirichlet series which satisfy a certain functional equation, Quart. Jour. of Math., Oxford series, $\mathbf{9}, 194$ to 195 (1938).

Los Angeles, January 11, 1950.

\title{
Forced Oscillations in Nonlinear Systems
}

\section{By Mary L. Cartwright}

\begin{abstract}
This paper shows how the approximate form of the solutions of a certain nonlinear differential equation occurring in radio work may be obtained from certain general results and gives the proof of the general results in detail. The proof of the general statement depends on a type of method that can be applied with minor modifications to any equation of the type

$$
\ddot{x}+k f(x) \dot{x}+g(x)=k p(t),
$$
\end{abstract}

where $p(t)$ has period $2 \pi / \lambda$, and $\int_{0}^{t} p(t) d t$ is bounded for all $t, f(x) \geq 1$ for $|x| \geq a$, and $g(x) / x \geq 1$ for $|x| \geq a$.

For some years Professor J. E. Littlewood and I have been working on nonlinear differential equations ${ }^{2}$ of a type which occur in radio work and elsewhere. One of the most interesting of these equations is

$$
\ddot{x}=k\left(1-x^{2}\right) \dot{x}+x-b k \lambda \cos (\lambda t+\alpha),
$$

especially for $k$ large and $0<b<2 / 3$. Our attention was drawn to it by a remark of van der $\mathrm{Pol},{ }^{3}$ which

1 This paper contains material presented in lecture form to the staff of the Institute for Numerical Analysis of the National Bureau of Standards on January 28,1949 . Miss Cartwright was a consultant at the INA at the time this lecture

was delivered.

2 See M. L. Cartwright and J. E. Littlewood, J. London Math. Soc. 20, 180
189 (1945), and Ann. Math. 48, 472-494 (1947); also M. L. Cartwright, J. Inst.

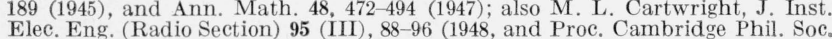
45, 495 (1949)

3 B. van der Pol, Proc. Inst. Radio Eng. 22, 1051-1086 (1934). suggested that it corresponded to a physical system investigated by him and van der Mark. ${ }^{4}$ For certain values of the parameters the physical system had two possible stable oscillations, one of period $4 n \pi / \lambda$ and one of period $(2 n+1) 2 \pi / \lambda$. As a matter of fact in the case of (1), owing to the strictly symmetrical nonlinear function $1-x^{2}$, the period $4 n \pi / \lambda$ does not occur, but for certain values of $b$ there are two stable oscillations of periods $(2 n \pm 1) 2 \pi / \lambda$.

It would take too long to give a complete proof of this statement here, but I propose to show how the approximate form of the solutions may be obtained from certain very general results, and give the proof of the general results in detail. The proof

4 B. van der Pol and J. van der Mark, Nature 120, 333-364 (1927) 
of the general statement depends on the type of method which we use throughout. It can be applied with minor modifications to any equation of the form

$$
\ddot{x}+k f(x) \dot{x}+g(x)=k p(t),
$$

where $p(t)$ has period $2 \pi / \lambda$, and $\int_{0}^{t} p(t) d t$ is bounded for all $t, f(x) \geq 1$ for $|x| \geq a$, and $g(x) / x \geq 1$ for $|x| \geq a$, provided of course that $f, g, p$ satisfy the usual conditions required for the existence and uniqueness of solutions, and are either independent of $k$ or satisfy certain simple inequalities independent of $k$.

We assume throughout that $k \geq 1$, and usually that it is large. We first observe that the $\alpha$ in (1) is merely inserted for convenience. It enables us to choose the origin of $t$ as we wish. We shall write $x_{o}, \dot{x}_{o}$ for the values of $x$ and $\dot{x}$ at $t=0$.

There are two main weapons of attack besides the equation (1) itself. The first is the integrated equation

$$
\begin{aligned}
& -\dot{x}-\dot{x}_{0}+k\left(\frac{x^{3}}{3}-x-\frac{x_{0}^{3}}{3}+x_{0}\right)+\int_{0}^{t} x d t \\
& =b k[\sin (\lambda t+\alpha)-\sin \alpha] ;
\end{aligned}
$$

the second is the energy equation which is obtained by multiplying (1) by $2 \dot{x}$ and integrating,

$$
\begin{aligned}
& \dot{x}^{2}-\dot{x}_{0}^{2}+2 k \int_{0}^{t}\left(x^{2}-1\right) \dot{x}^{2} d t+x^{2}-x_{0}^{2} \\
& =2 b k \lambda \int_{0}^{t} \dot{x} \cos (\lambda t+\alpha) d t .
\end{aligned}
$$

In some ways the integrated equation is more fundamental than (1), and that is why the $\lambda$ is inserted on the righthand side of (1). The energy equation may be rewritten with $\dot{x}=y, \dot{x}_{o}=y_{o}$ in the form

$$
\begin{aligned}
& x^{2}+y^{2}-\left(x_{0}^{2}+y_{0}^{2}\right) \\
& =2 k\left\{\int_{0}^{t} b \lambda \dot{x} \cos (\lambda t+\alpha)-\left(x^{2}-1\right) \dot{x}^{2}\right\} d t .
\end{aligned}
$$

The righthand side of $\left(4^{\prime}\right)$ will be dominated by the term $x^{2} \dot{x}^{2}$ when $x$ is large unless $\dot{x}$ is then very small, and it seems improbable that $\dot{x}$ can be small for most of the time that $x$ is large. This suggests that $x^{2}+y^{2}$ decreases rapidly over any arc for which $x$ is large, and therefore that $x$ is bounded for sufficiently large $t$. The general result which I propose to prove later is the following:

Theorem 1: If $x=x\left(t, x_{o}, \dot{x}_{o}\right)$ is any solution of (1) for which $x=x_{o}, \dot{x}=\dot{x}_{o}$ when $t=0$, then

$$
|x|<B,|\dot{x}|<B k \text {, }
$$

where $B$ is a constant independent of $k$ and $t$, for $k \geq 1$, $t>t_{o}\left(x_{o}, \dot{x}_{0}\right)$.
Assuming for the moment the result of theorem 1, we may argue as follows: eq 3 can be rearranged in the form

$$
\frac{x^{3}}{3}-x=b \sin (\lambda t+\alpha)+C-\frac{\dot{x}}{k}-\frac{1}{k} \int_{0}^{t} x d t,
$$

where $C$ is a constant depending on $x_{o}, \dot{x}_{o}, b$ and $\alpha$, and in virtue of (5) the last term is $O(1 / k)$ for large values of $k$ and $0 \leq t \leq 2 \pi / \lambda$. Hence there are two extreme possibilities: either $\dot{x}$ is comparable with $k$ in magnitude, or $x$ is given approximately by the equation $x=X$, where

$$
F(X)=\frac{X^{3}}{3}-X=b \sin (\lambda t+\alpha)+C .
$$

Both these possibilities may occur for arbitrarily large values of $t$, and also of course there are transitions from one to the other. For fixed $t$ and $b$ and $C$ such that $(-2 / 3)<b \sin (\lambda t+\alpha)+C<2 / 3$, eq 6 has three roots,

$$
X_{1}<-1<X_{2}<1<X_{3}=X_{3}(t, C),
$$

as may be seen from figure 1 . If $b>2 / 3$, $b \sin (\lambda t+\alpha)+C$ runs outside the interval $[-2 / 3,2 / 3]$ for some $t$ in each period; we suppose that $0<b<2 / 3$ because this is the most interesting range. It may be observed from figure 1 that if $X_{1}$ and $X_{3}$ increase with $t, X_{2}$ decreases and vice-versa, so the solutions over $0 \leq t \leq 2 \pi / \lambda$ are approximately as shown in figure 2 , provided that $|\dot{x}| \leq B$.

If we integrate over a second period $2 \pi / \lambda \leq t \leq 4 \pi$ $\lambda$, we have $\left(3^{\prime}\right)$ with $C^{\prime}$ in place of $C$ where

$$
\begin{aligned}
C^{\prime}=C-\frac{1}{k} \int_{2 \pi / \lambda}^{4 \pi / \lambda} x d t & =C-\frac{1}{k} \int_{2 \pi / \lambda}^{4 \pi / \lambda} X_{s}(t, C) d t+O\left(\frac{1}{k^{2}}\right), \\
s & =1,2,3 .
\end{aligned}
$$

Hence if $|\dot{x}|<B$, the solution is given approximately by $X_{s}\left(t, C^{\prime}\right)$ over the next period. The difference $C^{\prime \prime}-C$ is actually of the same order as $\dot{x} / k$, which occurs in the error term in $\left(3^{\prime}\right)$, but it can be shown that in the circumstances considered $\dot{x}$ is of the form $\dot{x}_{s}(t, C)+O\left(k^{-1 / 2}\right)$. Putting this and $x=X_{s}(t, C)+\mathrm{O}\left(k^{-1}\right)$ in $\left(3^{\prime}\right)$, and remembering that $X_{s}$ and $\dot{x}_{s}$ are periodic, we see than the nonperiodic error is

$$
\frac{1}{k} \int_{0}^{t} X_{s}(t, C) d t+O\left(\frac{1}{k^{3}}\right)
$$

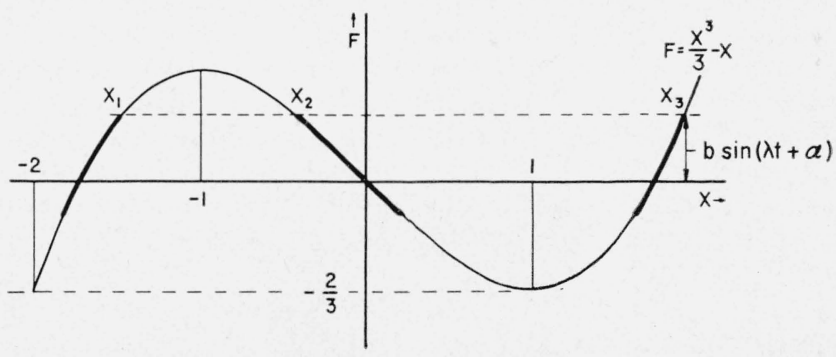

Figure 1. 


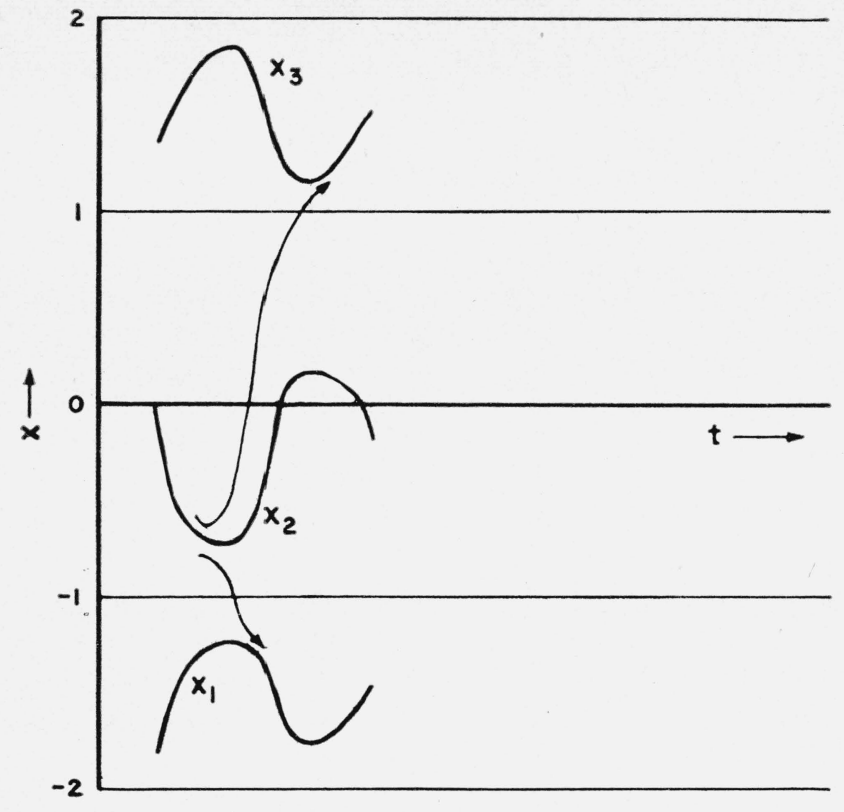

Figure 2.

and so the second wave will certainly be lower than the first in the case of $X_{3}$ by an amount of order $1 / k$, and the solution will perform descending waves as shown in figure 3 . The second wave will be higher than the first in the case of $X_{1}$, and higher or lower for $X_{2}$, unless

$$
\int_{0}^{2 \pi / \lambda} X_{2}(t, C) d t=0
$$

(so that $C^{\prime}=C$ approximately). This can only occur for $C=0$, and then we have a solution with period $2 \pi / \lambda$ approximately. There is in fact a strictly periodic solution with period $2 \pi / \lambda$ which is, for reasons which we shall give presently, unstable.

Returning to the case in which $|\dot{x}|$ is comparable with $k$ in magnitude, we suppose first that $\dot{x}>\delta k$, where $\delta>0$ for a time of length $D / k$. Then $x$ changes by at least $D \delta$, and this is excluded by (5) if $D \delta>2 B$. Similarly $\dot{x}$ is not less than $-\delta k$ for an interval of time greater than $D / k$, for if it is $x$ runs outside the strip $|x|<B$.

As regards the transitions, if $\left|1-x^{2}\right|>\delta>0$ and $|\dot{x}|$ is large, the second term in (1) is much more important than $x$ or $b k \lambda \cos (\lambda t+\alpha)$, and so $\ddot{x}$ has the same sign as $\dot{x}$ in $|x|<1-\delta$ and the opposite if $|x|>1+\delta$, (provided that $|\dot{x}|$ is large and $k$ is large). Hence any small but significant deviation from $X_{1}$ or $X_{3}$ is at once corrected, whereas a small but signicant deviation from $X_{2}$ causes $|\dot{x}|$ to increase rapidly. In the latter case $|\dot{x}|$ cannot decrease again until $x$ approaches either the $X_{1}$ or $X_{3}$ curve which corresponds.

Finally near $x= \pm 1$ the term $b k \lambda \cos (\lambda t+\alpha)$ dominates except when $\lambda t+\alpha$ is near $n \pi+1 / 2 \pi$. The dips of $X_{3}$ towards $x=1$ and crests of $X_{1}$ toward $x=-1$ do in fact occur in these intervals, and a complete theory of the behavior of solutions near these points is very complicated. However, in spite of that we can now form a fairly good general picture of the behavior of solutions. They settle above $x=1$ into a long descent in waves of the form $x=X_{3}(t, C)$ approximately, dipping a little lower each time until they reach the neighborhood of $x=1$. There they have three alternatives: (1) another wave $x=X_{3}$ just above $x=1,(2)$ an unstable wave $x=X_{2}$ just below $x=1$, or (3) a rush down to the corresponding $X_{1}$ which, as may be seen from figure 1 , is near $x=-2$. The stable oscillations naturally follow the first or third alternative, and have period $(2 n+1) 2 \pi / \lambda$ or $(2 n-1) 2 \pi / \lambda$ according as they have $n+1 / 2$ or $n-1 / 2$ waves above $x=1$. Separating them there are many types of unstable motion following an $X_{2}$ curve which begins and ends on $x=1$ for part or the whole of the way (or a similar $X_{2}$ curve after a long ascent near $x=-1$ ). From an $X_{2}$ curve they may pull up sharply to $X_{1}$ or rush down to $X_{3}$ at any stage. All types starting near $x=1$ finally plunge down to the $X_{1}$ curve, and perform a corresponding long ascent to $x=-1$, the whole phenomenon for $x<0$ corresponding to that for $x>0$. All this can be rigorously established, and moreover the relative positions of solutions approximating to $X_{3}\left(t, C_{1}\right)$ and $X_{3}\left(t, C_{2}\right)$ remain the same throughout a long descent unless $C_{1}$ and $C_{2}$ differ by something which is extremely small for large $k$, such as $e^{-k^{3 / 2}}$.

We now return to the proof of theorem 1 . The preceding analysis depended to a large extent on the fact that $1-x^{2}$ changes sign twice; in the work which follows the significant point is that the coefficient of $x$ is positive for large $x$ and that the function $g(x)$ in (2) has the sign of $x$. Physicists may consider it intuitive that a system with a restoring force and positive damping for large $x$ should have bounded solutions, and I hope to show that this is also intuitive by mathematical commonsense. Incidentally the lemmas give a good deal of other information about the solutions. I shall as usual refer to $t$ as the time, and say that $t_{1}$ is before or after $t_{2}$ according as $t_{1}<t_{2}$ or $t_{1}>t_{2}$. The constants $B$ are independent of $k$ and $t$, and are not necessarily the same in each place unless a suffix is attached, and, as we said earlier $k>1$.

We first ensure that all solutions eventually come fairly near $x=0$.

Lemma 1. A solution of (1) cannot have absolute value greater than $3^{1 / 2}$ for all large $t$.

Suppose that this is not so, and that $x \geq 3^{1 / 2}$ for $t \geq t_{1}$. Integrating from $t_{1}$ where $x=x_{1}, \dot{x}=\dot{x}_{1}$, we have

$\dot{x}-\dot{x}_{1}+k\left(\frac{x^{3}}{3}-x-\frac{x_{1}^{3}}{3}+x_{1}\right)+\int_{t_{1}}^{t} x d t=b k[\sin (\lambda t+\alpha)-\sin \alpha]$,

and so

$$
3^{1 / 2}\left(t-t_{1}\right) \leq \int_{t_{1}}^{t} x d t=-\dot{x}+k x\left(1-\frac{x^{2}}{3}\right)+O(1)
$$

as $t \rightarrow \infty$, where the constant implied depends on $k$. The lefthand side tends to $\infty$, and so $x \rightarrow-\infty$, but this implies that $x \rightarrow-\infty$, which contradicts $x \geq 3^{1 / 2}$. Hence $x \leq 3^{1 / 2}$ for arbitrarily large $t$, and similarly $x \geq-3^{1 / 2}$ for some large $t$. 
We next establish that as long as $|x|$ is not too large the value of $|\dot{x}|$ cannot increase too much.

Lemma 2. If $|x| \leq 3^{1 / 2}$ on an arc $P Q$, then

$$
\left|\dot{x}_{Q}\right|<\left|\dot{x}_{P}\right|+B_{1} k,
$$

and, if the arc lasts a time longer than $4 / k \leq 4,\left|\dot{x}_{Q}\right| \leq 2$. More generally if $|x| \leq b_{o}$ on an arc $P Q$, then

$$
\left|\dot{x}_{Q}\right|<\left|\dot{x}_{P}\right|+B_{1}\left(b_{o}\right) k .
$$

If $|x| \leq 3^{1 / 2}$ and $\dot{x}>k$ on an arc, it can only last a time at most $2.3^{1 / 2} / k<4 / k$. For if it lasts longer, the solution travels a distance more than $2.3^{1 / 2}$ and therefore cannot remain in the strip. The same is true if $\dot{x}<-k$ on an arc in $|x| \leq 3^{3 / 2}$.

Let $P_{1}$ be the last point before $Q$ at which $|\dot{x}| \leq k$, or $P$ itself, whichever is the latest. Suppose first that $\dot{x}_{P_{1}}>0$, then $\dot{x}>0$ on the arc $P_{1} Q$. For, if not, $\dot{x}=0$ at some point of $P_{1} Q$ and $P_{1}$ is not the last point at which $|\dot{x}| \leq k$. By the integrated equation, since $\left|x_{\mathrm{Q}}\right|<3^{1 / 2},\left|x_{P_{1}}\right|<3^{1 / 2}$,

$$
\dot{x}_{Q}-\dot{x}_{P_{1}} \leq B_{1} k-\int_{t_{1}}^{t} x d t \leq B_{1} k,
$$

where $B_{1} \geq 1$ depends on $b$, which gives the result. Similarly if $\dot{x}_{P_{1}}<O$, we have $\dot{x}_{P_{1}}-\dot{x}_{\mathrm{Q}} \leq B_{1} k$. If the time is greater than $4 k,\left|\dot{x}_{P_{1}}\right| \leq k$, and we have the second form of the result. The result for $|x| \leq b_{o}$ follows by the same method.

The next two lemmas show that the height of an arc $Q R$ outside the strip $|x| \leq 3^{1 / 2}$, and the time taken to describe it are bounded by numbers depending on $\dot{x}_{Q}$; in other words the velocity with which it emerges from the strip.

Lemma 3. If the arc $Q R$ lies above $x=3^{1 / 2}$ and begins at $Q$ on $x=3^{1 / 2}$, the greatest height h satisfies

$$
h<\frac{\dot{x}_{Q}}{2 k}+B_{2} \text {. }
$$

When $x=h=3^{1 / 2}+h_{1}, \dot{x}=0$. Integrating from the point $Q$ to the point $H$ at which $x=h$, we have

$$
\begin{aligned}
0=\dot{x}_{Q} & -k \int_{3^{3 / 2}}^{3^{3 / 2}+h_{1}}\left(x^{2}-1\right) d x \\
& -\int_{0}^{t} x d t+b k[\sin (\lambda t+\alpha)-\sin \alpha] .
\end{aligned}
$$

Since $x^{2}-1 \geq 2$ on $Q R$,

$$
0<\dot{x}_{Q}-2 k h_{1}+B k
$$

and the result follows for $h_{1}$ and so for $h$.

Lemma 4. If $Q R$ is an arc above $x=3^{1 / 2}$ beginning at $Q$ on $x=3^{3 / 2}$, then the time t taken to describe $Q R$ is less than $B_{3} \dot{x}_{Q}$, provided that $\dot{x}_{Q}>k$.
Suppose that $\dot{x}_{Q}>k$, and that $R$ is on $x=3^{1 / 2}$ so that $x_{Q}=x_{R}=3^{1 / 2}$. On $Q R$ it is easy to see that

$$
\begin{gathered}
\dot{x}<\dot{x}_{Q}+B k-\int_{0}^{t} x d t \\
<B \dot{x}_{Q}-3^{1 / 2} t
\end{gathered}
$$

because $\dot{x}_{Q}>k$. Also

$$
0=x_{R}-x_{Q}=\int_{0}^{t} \dot{x} d t<B \dot{x}_{Q} t-\frac{3^{1 / 2}}{2} t^{2}
$$

and so $t<B_{3} \dot{x}_{Q}$.

The reduction in energy over any sufficiently high arc is established in lemma 5 which gives effect to the remarks we made about $\left(4^{\prime}\right)$. It is much the most difficult part of the proof.

Lemma 5. If $Q R$ is an arc above $x=3^{1 / 2}$ beginning at $Q$ on $x=3^{1 / 2}$ and ending at $R$ on $x=3^{1 / 2}$, for a given $B_{1} \geq 1$ there exists a $B_{4} \geq B_{1} \geq 1$ such that if $\dot{x}_{Q}>B_{4} k$,

$$
\dot{x}_{R}^{2}<\dot{x}_{Q}^{2}-4 B_{1} k \dot{x}_{Q} .
$$

The energy eq 4 for the arc $Q R$ is

$$
\dot{x}_{R}^{2}-\dot{x}_{Q}^{2}=-2 k \int_{0}^{t}\left(x^{2}-1\right) \dot{x}^{2} d t+2 b k \lambda \int_{0}^{t} \dot{x} \sin (\lambda t+\alpha) d t .
$$

Let

Then

$$
J=k \int_{0}^{t}\left(x^{2}-1\right) \dot{x}^{2} d t \geq k \int_{0}^{t} \dot{x}^{2} d t .
$$

$$
\begin{aligned}
& \dot{x}_{R}^{2}-\dot{x}_{Q}^{2} \leq-2 J+2 b k \lambda \int_{0}^{t}|\dot{x}| d t \\
& \leq-2 J+B k t^{3 / 2}\left(\int_{0}^{t}|\dot{x}|^{2} d t\right)^{1 / 2}
\end{aligned}
$$

by Cauchy's inequality. So by lemma 4 and (8)

$$
\begin{gathered}
\dot{x}_{R}^{2}-\dot{x}_{Q}^{2} \leq-2 J+B_{5} k^{1 / 2} \dot{x}_{Q}^{1 / 2} J^{1 / 2} \\
\leq-2 J\left[1-\frac{1}{2} B_{5}\left(\frac{k \dot{x}_{Q}}{J}\right)^{1 / 2}\right] \\
<-J<-B_{5}^{2} k \dot{x}_{Q},
\end{gathered}
$$

provided that $J \geq B_{5}^{2} k \dot{x}_{Q}$, where $B_{5}$ depends on $B_{3}$ and $\dot{x}_{Q} \geq k$. We may obviously choose $B_{5}^{2}>4 B_{1}$, and we have the result except for the case in which $J<B_{5}^{2} k \dot{x}_{Q}$.

Suppose now that $J<B_{5}^{2} k \dot{x}_{Q}$, and integrate from $Q$ until $\dot{x}=\frac{1}{2} \dot{x}_{Q}$ or $x=2 B_{5}^{2}+3^{1 / 2}$, whichever comes first. Then $\dot{x} \geq \frac{1}{2} \dot{x}_{Q}>0$. Hence $x$ is increasing on this are and

$\int_{0}^{t} \sin (\lambda t+\alpha) \dot{x} d t=\int_{3^{1 / 2}}^{x} \sin (\lambda t+\alpha) d x<\int_{3^{1 / 2}}^{x} d x \leq 2 B_{5}^{2}$. 
Hence

$$
\begin{gathered}
\dot{x}^{2}-\dot{x}_{Q}^{2}=-2 k \int_{0}^{t}\left(x^{2}-1\right) \dot{x}^{2} d t-k b \lambda \int_{0}^{x} \sin (\lambda t+\alpha) d x-B \\
\geq-2 J-k B B_{5}^{2}-B \\
\geq-2 k B_{5}^{2} \dot{x}_{Q}-k B \geq-\frac{3}{4} \dot{x}_{Q}^{2},
\end{gathered}
$$

provided that $\dot{x}_{Q}>k B_{4}$. It follows that $\dot{x}^{2}>\frac{1}{4} \dot{x}_{Q}^{2}$ and therefore $\dot{x}>\frac{1}{2} \dot{x}_{Q}$, so that $x$ reaches $2 B_{5}^{2}+3^{1 / 2}$ first. Now since $x^{2}-1 \geq 2$ on the arc,

$$
J \geq 2 k \int_{3^{1 / 2}}^{2 B_{5}^{2}+3^{1 / 2}}|\dot{x}| d x \geq k \dot{x}_{Q} \cdot 2 B_{5}^{2},
$$

and we have a contradiction. Hence the result of the lemma is true.

It remains to combine the results of lemmas 2 and 5 .

Lemma 6. If $Q$ is a point on $x=3^{1 / 2}$ such that $\dot{x}_{Q}>B_{4} k>0$, then the solution returns to the strip $|x| \leq 3^{1 / 2}$ at $R$ and emerges again at $S$ with $\left|\dot{x}_{S}\right|<\left|\dot{x}_{Q}\right|$ $-\bar{B}_{1} k$.

By lemma 2 with $R$ and $S$ in place of $P$ and $Q$

$$
\left|\dot{x}_{S}\right|^{2}<\left|\dot{x}_{R}\right|^{2}+2 B_{1} k\left|\dot{x}_{R}\right|+B_{1}^{2} k^{2} .
$$

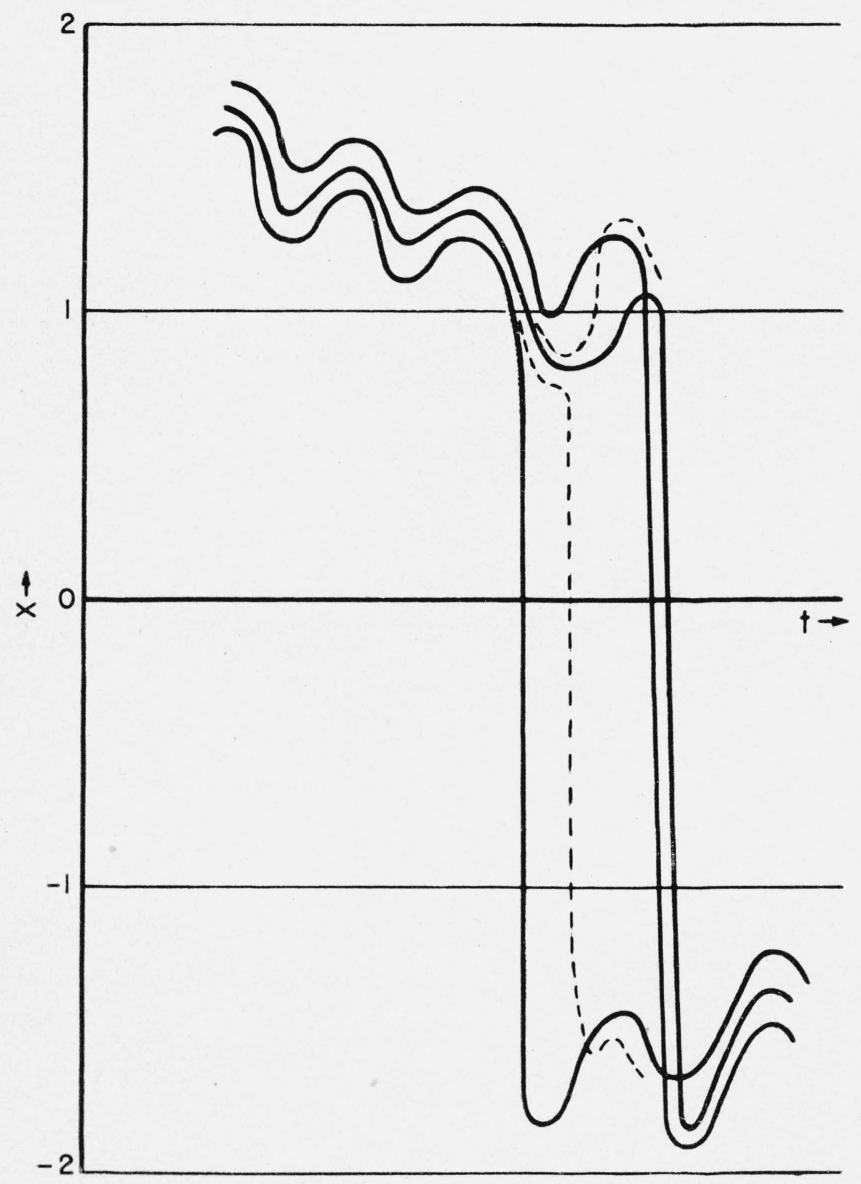

Figure 3.
Using lemma 5 in the form stated and also in the weaker form $\left|\dot{x}_{R}\right| \leq\left|\dot{x}_{Q}\right|$, we have

$$
\begin{gathered}
\left|\dot{x}_{S}\right|^{2}<\left|\dot{x}_{Q}\right|^{2}-4 B_{1} k\left|\dot{x}_{Q}\right|+2 B_{1} k\left|\dot{x}_{R}\right|+B_{\mathrm{I}}^{2} k \\
=\left(\left|\dot{x}_{Q}\right|-B_{1} k\right)^{2},
\end{gathered}
$$

From which the result follows. For $\dot{x}_{Q}>B_{4} k \geq B_{1} k$.

We now have the result stated in theorem 1 . For by lemma 1 the solution must enter the strip $|x| \leq 3^{3 / 2}$, and by the second part of lemma $2|\dot{x}| \leq B_{1} k$, if it stays there. If on the other hand it emerges at $Q$ with $\left|\dot{x}_{Q}\right| \leq B_{4} k$, the height (or depth) of the subsequent arc outside the strip is less than $B_{4}+B_{2}$. For by symmetry all the lemmas for $x-\geq 3^{\frac{1}{2}}$ have strictly corresponding forms for $x \leq-3^{1 / 2}$. But by lemma 6 if $\left|\dot{x}_{Q}\right|>B_{4} k$, the solution emerges the next time at $S$ with $\left|\dot{x}_{S}\right|<\left|\dot{x}_{Q}\right|-B_{1} k$, so that it must either stay in the strip or emerge eventually with $\left|\dot{x}_{Q}\right| \leq B_{4} k$, and then by the second part of lemma $2,|x| \leq \overline{B_{4}}+B_{2}$ for all subsequent $t$.

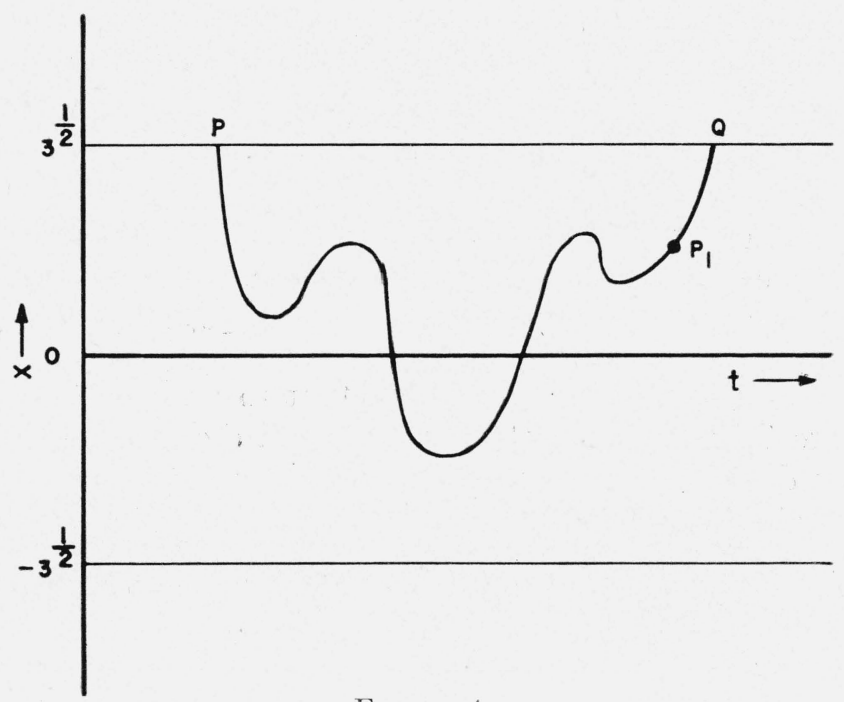

FIGURE 4.

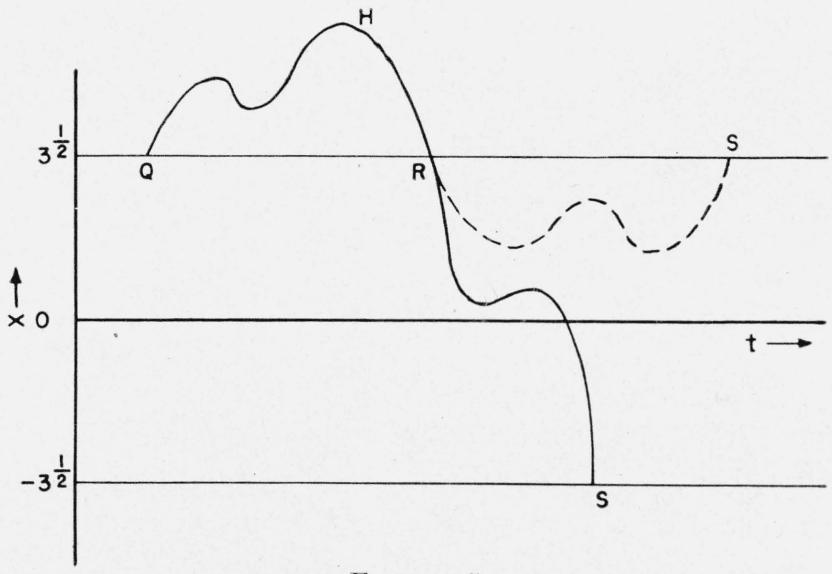

Figure 5.

Los Angeles, December 16, 1949. 